\title{
Método Craneométrico de Knebelman: Modificación clínica para simplificar la determinación de la Dimensión Vertical Oclusal.
}

\section{Knebelman craniometric method: clinical modification to simplify the determination of the Vertical Occlusal Dimension.}

\begin{abstract}
Marcelo Gaete-Baldi1${ }^{*}$, Matías Muñoz-Olavarría²
1. Clínica Integral del Adulto y Odontogeriatría, Universidad Andrés Bello, Santiago, Chile.

2. Universidad Andrés Bello, Santiago, Chile.

* Correspondencia autor. Marcelo Gaete-Baldi. | Manquehue Sur 520 Of. 416 Las Condes | Teléfono: +56998940523 | E-mail: gaetebaldi@ hotmail.com

Trabajo recibido el 11/11/2018.

Aprobado para su publicación el 14/12/2018

\section{RESUMEN}

Objetivo: Determinar la relación entre la distancia clínica Ojo / Tragus y la distancia craneométrica Ojo / Oreja. Materiales y Métodos: En un grupo de 100 individuos, 50 hombres y 50 mujeres, se realizaron dos medidas. Distancia desde pared anterior del canal auditivo externo a la esquina lateral de la órbita con el craneómetro de Knebelman y distancia surco tragus facial al ángulo externo del ojo con un pie de metro digital. A las medidas se les realizó análisis estadístico descriptivo y de Concordancia Bland \& Altman. Resultados: Las distancias presentaron una diferencia promedio de $-0,761$ $\mathrm{mm}$. en el total, $-0,670 \mathrm{~mm}$. en hombres, $-0,852 \mathrm{~mm}$. en mujeres. El análisis estadístico, arrojó concordancia solo en hombres, el análisis de la magnitud de sobre estimación estableció $2,34 \%$ en el total y $0,23 \%$ en mujeres. Con estos datos se modificó el factor de ajuste proporcional del método original. Conclusión: No existe una concordancia perfecta entre ambas distancias, pero el análisis de las sobre estimaciones nos permite modificar el método de Knebelman para su aplicación clínica sin Craneómetro, estableciendo que la distancia clínica surco tragus facial al ángulo externo del ojo menos $5 \mathrm{~mm}$ nos permite evaluar o establecer la Dimensión Vertical Oclusal.
\end{abstract}

PALABRAS CLAVE:

Dimensión vertical oclusal; Craneómetro; Knebelman.

Rev. Clin. Periodoncia Implantol. Rehabil. Oral Vol. 12(1); 27-30, 2019.

\section{ABSTRACT}

Objective: to determine the relationship between the clinical distance Eye / Tragus and the cranial distance Eye / Ear. Materials and Methods: In a group of 100 individuals, 50 men and 50 women, two measurements were made. Distance from the anterior wall of the external auditory canal to the lateral corner of the orbit with the Knebelman craniometer and distance from the facial tragus furrow to the external angle of the eye with a digital meter foot. A descriptive and concordance statistical analysis of Bland \& Atlman was carried out on these measures. Results: The distances showed a mean difference of $-0.761 \mathrm{~mm}$. in the total, $-0.670 \mathrm{~mm}$. in men, $-0,852 \mathrm{~mm}$. in women. Statistical analysis was concordant only in men. The magnitude analysis of overestimate established $2.34 \%$ in the total and $0.23 \%$ in women. With these data, the proportional adjustment factor of the original method was modified. Conclusion: There is no perfect agreement between the two distances, but the overestimates analysis allows us to modify the Knebelman method for its clinical application without Craniometer, establishing that the clinical distance from the facial tragus furrow to the external angle of the eye minus 5 $\mathrm{mm}$. allows us to evaluate or establish the Vertical Occlusal Dimension..

KEY WORDS:

Vertical occlusal dimension; Craniometer; Knebelman.

Rev. Clin. Periodoncia Implantol. Rehabil. Oral Vol. 12(1); 27-30, 2019. 


\section{INTRODUCCIÓN}

En Rehabilitación Oral se deben cumplir varias etapas clínicas y de laboratorio para asegurar el éxito del tratamiento. Una de las etapas que adquiere mayor relevancia, es la determinación de las Relaciones Cráneo Mandibulares. A nivel de la articulación temporomandibular se observa que a una correcta relación vertical el cóndilo se encuentra en relación céntrica, es decir, la relación vertical influencia a la horizontal. Estas variaciones fisiológicas de la posición mandibular, en especial en el plano vertical, se pueden cuantificar y medir a través de la Dimensión Vertical, definida como la altura del tercio inferior del rostro determinada entre dos puntos arbitrariamente seleccionados y convencionalmente localizados, uno en el maxilar (nasal o subnasal) y el otro en la mandíbula (mentón), coincidentes en la línea media ${ }^{(1,2)}$.

Una Dimensión Vertical aumentada o disminuida, afecta tanto a la estética facial como a la función del sistema estomatognático ${ }^{(3,4,5)}$, razón por la cual su determinación implica una etapa de gran importancia para el correcto funcionamiento y homeostasis del sistema.

Se han investigado diversos métodos para determinar la dimensión vertical ${ }^{(6,7,8,9,10)}$, signo claro del interés que despierta este tema, junto con revelarnos que estamos todavía muy lejos de obtener el método ideal. Estos métodos fueron clasificados por Carl Misch ${ }^{(11)}$ en métodos subjetivos y objetivos. Los métodos subjetivos son aquellos métodos que tienen una alta variabilidad o que están sujetos a un sin número de agentes condicionantes, ya que para determinar la dimensión vertical utilizan acciones fisiológicas del paciente y requieren del juicio y/o preferencias del clínico(9). Los métodos objetivos son aquellos métodos que basan sus resultados en proporciones y medidas realizadas en referencias anatómicas del paciente. Por lo tanto, se supone, que arrojan resultados más exactos en los que no influye el carácter emocional en que se encuentre el paciente y reduce la percepción subjetiva del clínico ${ }^{(9)}$.

Dentro de estos últimos encontramos el método Craneométrico de Knebelman ${ }^{(7,12)}$, quien plantea que en cráneos donde el crecimiento, desarrollo y oclusión son normales, es posible correlacionar distancias de marcas craneofaciales y registrar una medición desde el cráneo que puede ser usada para ayudar a establecer la Dimensión Vertical Oclusal (DVO). Este método establece que la distancia desde la pared anterior del canal auditivo externo a la esquina lateral de la órbita (distancia OJO/ OREJA) está proporcionalmente relacionada con la distancia entre el mentón (superficie inferior más anterior de la mandíbula) y la espina nasal.

Diversos autores $(7,9,10,13,14,15,16,17)$ han establecido que el método Craneométrico de Knebelman, es un método diagnóstico para la determinación y evaluación de la DVO. Los resultados obtenidos han establecido que la distancia OJO/OREJA puede ser utilizada para predecir la DVO con razonable seguridad y promueven al craneómetro como un instrumento de gran utilidad en la práctica clínica diaria, pues es un método simple, de fácil aprendizaje y poco invasivo. Sin embargo, este último hecho es su mayor inconveniente, ya que se requiere de la adquisición de este dispositivo.

Romo ${ }^{(8)}$ traspasó los puntos de la primera medida de Knebelman a la piel e investigó si la distancia clínica ángulo externo de ojo / surco tragus facial (distancia OJO/TRAGUS) era igual a la DVO, concluyendo que esta distancia clínica puede ser utilizada para ayudar a determinar la DVO, fundamentalmente en individuos mesofaciales.

Partiendo de la base que diversos autores han establecido que el método Craneométrico de Knebelman, es un método válido para evaluar o determinar la DVO, el objetivo del presente estudio es determinar la relación entre la distancia clínica OJO/TRAGUS medida con un pie de metro y la distancia craneométrica OJO/OREJA medida con el Craneómetro de Knebelman.

\section{MATERIAL Y MÉTODO}

Materiales: Pie de metro digital DIN 862 Stainless Hardened, Craneómetro de Knebelman, 1 frasco de algodón, 1 litro de alcohol y 1 plumón negro de punta fina. Selección de la muestra: Del universo disponible, se seleccionó una muestra de 100 alumnos estudiantes de odontología de la Universidad Andrés Bello, Santiago. 50 hombres y 50 mujeres, todos adultos jóvenes. Para seleccionar la muestra se utilizó un método de muestreo no probabilístico, por conveniencia. Los individuos seleccionados poseen dentición natural, ausencia de tratamiento de ortodoncia previo o en curso y no tienen pérdida ni alteración de la mesa oclusal posterior por pérdida de dientes. Individuos con características clínicas evidentes de Clase III esqueletal y asimetrías faciales evidentes fueron eliminados de la muestra.

\section{Procedimiento Clínico:}

1.- Medición distancia pared anterior del canal auditivo externo
I borde lateral de la órbita. (Distancia OJO/OREJA). El individuo fue sentado cómodamente con su espalda apoyada y su cabeza en posición natural. Con el Craneómetro de Knebelman (Figura 1) se realizó la medición colocando la punta para el conducto auditivo externo dentro de meato derecho, con la punta cómodamente insertada dentro del meato, se deslizó la punta orbitaria hasta alcanzar el borde lateral de la órbita ocular. Con ambas puntas colocadas en posición y sin causar ningún tipo de molestia, se asegura firmemente el tornillo de fijación. (Figura 2A)

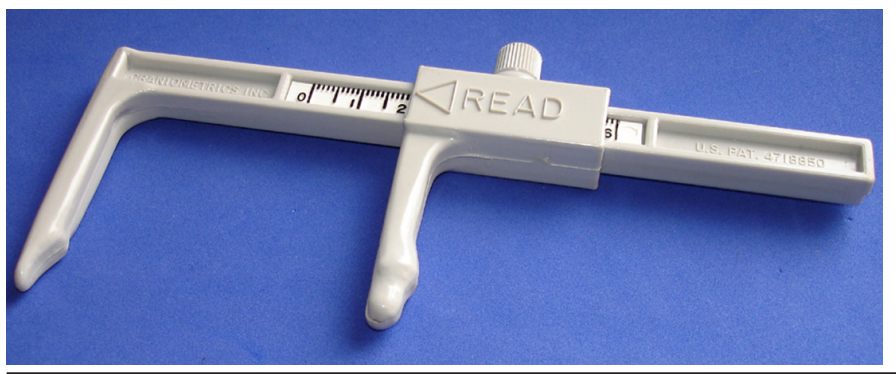

Figura 1. Craneómetro de Knebelman

2.- Medición distancia clínica ángulo externo del ojo / tragus facial. (Distancia OJO/TRAGUS). El individuo fue sentado cómodamente con su espalda apoyada y su cabeza en posición natural. En esa posición se marcó en el lado derecho un punto en el tragus con un plumón negro de punta fina, el cual, se localizó mediante la línea de reflexión de la piel, dónde el tragus termina y comienza la piel del rostro frente al conducto auditivo externo, coincidente con el vértice del tragus (Figura 3). La distancia desde este punto del tragus hasta el ángulo externo del ojo se midió con un pie de metro digital sin comprimir los tejidos. (Figura 2B)
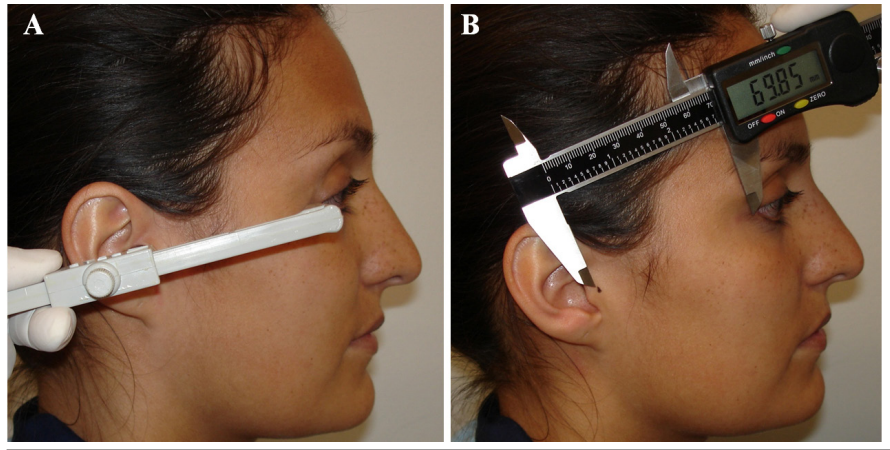

Figura 2. A.- Medición distancia OJO/OREJA B.- Medición distancia OJO/TRAGUS
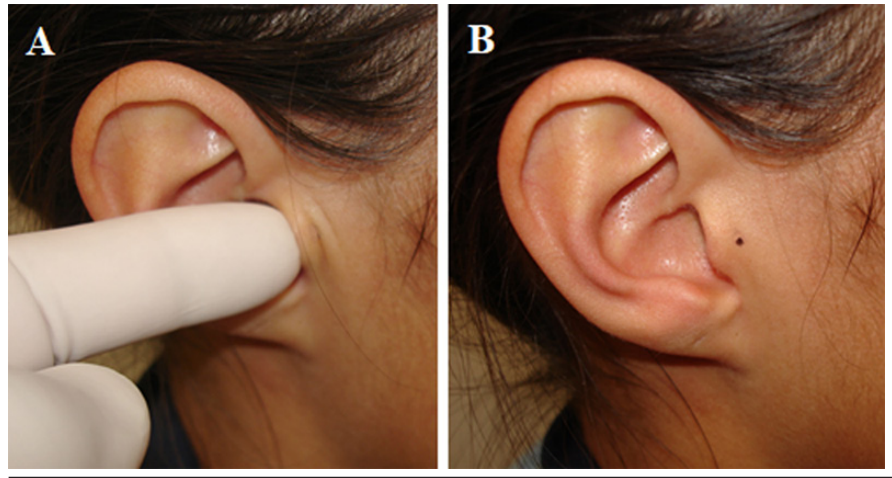

Figura 3. Determinación del punto Surco Tragus Facial

Ambas distancias fueron cuantificadas en milímetros, anotadas en una ficha individual para cada participante, tabuladas en Excel y analizadas estadísticamente mediante el software Stata 14.2, tanto en el grupo total, como por género. Mediante estadística descriptiva se estableció el promedio, desviación estándar, valores máximos y mínimos. La concordancia se analizó mediante el Análisis Estadístico de Concordancia de Bland \& Altman. Con el objeto de evitar al máximo los errores, ambas mediciones fueron realizadas por un solo operador, 
el cual se calibró realizando previamente solo las mediciones siguiendo los protocolos de Knebelman ${ }^{(12)}$ y Romo(8), estas mediciones fueron chequeadas por un examinador con mayor experiencia, cuando las medidas no eran coincidentes se revisaron los protocolos y se volvió a medir.

Las mediciones se realizaron en el lado derecho con la finalidad de comparar nuestros resultados con los de $\operatorname{Romo}^{(8)}$ y Contreras ${ }^{(18)}$, sin embargo, Gaete ${ }^{(7)}$ determinó que si bien no existen diferencias estadísticamente significativas entre el lado derecho e izquierdo, los valores del lado derecho se acercaban más a los de DVO. También el protocolo original de Knebelman ${ }^{(12)}$ plantea realizar la medición en el lado derecho y si el operador tiene dudas medir el lado izquierdo, de ser valores diferentes debe utilizar el promedio.

\section{RESULTADOS}

La estadística descriptiva (Tabla I) nos muestra una diferencia promedio entre ambas distancias de $-0,76 \mathrm{~mm}$. $\pm 0,35$ en el grupo total, de $-0,67 \mathrm{~mm}$. $\pm 0,36$ en el grupo de hombres y de $-0,85 \mathrm{~mm}$. $\pm 0,31$ en el grupo de las mujeres.

Tabla 1. Análisis estadístico descriptivo del grupo total, hombres y mujeres

\begin{tabular}{|cccc|}
\hline VARIABLE & $\begin{array}{c}\text { PROM./D.S. } \\
\text { TOTAL } \\
(\mathbf{N}=\mathbf{1 0 0})\end{array}$ & $\begin{array}{c}\text { PROM./D.S. } \\
\text { HOMBRES } \\
(\mathbf{N}=\mathbf{5 0})\end{array}$ & $\begin{array}{c}\text { PROM./D.S. } \\
\text { MUJERES } \\
(\mathbf{N}=\mathbf{5 0})\end{array}$ \\
\hline Dist. $\mathbf{O j} / \mathbf{O r}$ & $72,3 \pm 3,79$ & $74,54 \pm 2,90$ & $70,06 \pm 3,23$ \\
\hline Dist. $\mathbf{O j}$ /Tr & $\mathbf{7 3 , 0 6 \pm 3 , 7 2}$ & $75,21 \pm 2,84$ & $70,91 \pm 3,24$ \\
\hline & & & \\
Diferencia & $\mathbf{- 0 , 7 6} \pm \mathbf{0 , 3 5}$ & $\mathbf{- 0 , 6 7} \pm \mathbf{0 , 3 6}$ & $\mathbf{- 0 , 8 5} \pm \mathbf{0 , 3 1}$ \\
\hline
\end{tabular}

El análisis estadístico de Concordancia de Bland \& Altman reveló que sistemáticamente la distancia OJO/TRAGUS sobre estima a la distancia OJO/OREJA, encontrando concordancia entre ambas medidas solo en el grupo de los hombres (Tabla II)

Tabla 2. Análisis Estadístico de Concordancia de Bland \& Altman

\begin{tabular}{|cccccc|c|c|}
\hline Grupo & N & Dif. Prom. & D.S. & $95 \%$ Límites de Concordancia & \multicolumn{2}{c|}{ Conc. } \\
\hline Total & 100 & -0.761 & 0.354 & -1.454 & -0.068 & No \\
\hline Hombres & 50 & -0.670 & 0.368 & -1.392 & 0.052 & Si \\
\hline Mujeres & 50 & -0.852 & 0.316 & -1.472 & -0.232 & No \\
\hline
\end{tabular}

En los grupos no concordantes se realizó el análisis de la magnitud de la sobre estimación (Tabla III), determinándose que la distancia OJO/ TRAGUS sobre estima a la distancia OJO/OREJA en un $2,34 \%$ en el grupo total y en solo un $0,23 \%$ en el grupo de las mujeres.

Tabla 3. Análisis de la magnitud de la sobre estimación

\begin{tabular}{|ccccccc|}
\hline Total & Coef. & Std. Err. & $t$ & P>t & $95 \%$ Intervalo de Confianza \\
\hline Oj/Tr & 1,0234 & 0,0091 & 107,31 & 0 & 1,0056 & 1,04124 \\
& & & & & & \\
\hline Oj/Or & 2,4538 & 0,6589 & 3,72 & 0 & 1,1464 & 3,76133 \\
& & & & & & \\
\hline Mujeres & Coef. & Std. Err. & $t$ & P>t & $95 \%$ Intervalo de Confianza \\
\hline Oj/Tr & 1,0023 & 0,0141 & 70,78 & 0 & 0,9747 & 1,0300 \\
\hline Oj/Or & 1,0150 & 0,9886 & 1,0300 & 0,3100 & $-0,9726$ & 3,0027 \\
\hline
\end{tabular}

Rev. Clin. Periodoncia Implantol. Rehabil. Oral Vol. 12(1); 27-30, 2019 | 29

\section{DISCUSIÓN}

Muchos son los métodos que se han estudiado para la determinación de la Dimensión Vertical. El método Craneométrico de Knebelman ha demostrado ser un método predictivo objetivo, simple, de fácil aprendizaje, poco invasivo y que determina específicamente la $D \mathrm{O}^{(7,9,10,13,14,15,16,17)}$, sin embargo requiere de la adquisición de un dispositivo especial para realizar las medidas. Evaluamos la distancia facial ángulo externo del ojo al surco tragus facial propuesta por $\mathrm{Romo}^{(8)}$ como alternativa a la distancia pared anterior del canal auditivo externo al borde lateral de la órbita que se debe determinar con el Craneometro, con la finalidad de poder aplicar el Método Craneométrico de Knebelman sin la necesidad de tener el dispositivo.

La estadística descriptiva de nuestros resultados nos muestra que la distancia OJO/TRAGUS es mayor que la distancia OJO/OREJA, tanto en el grupo total en estudio como por género. Observando una diferencia promedio entre ambas distancias de $-0,76 \mathrm{~mm}$. $\pm 0,35$ en el grupo total, de $-0,67 \mathrm{~mm}$. $\pm 0,36$ en el grupo de hombres y de $-0,85 \mathrm{~mm} . \pm 0,31$ en el grupo de las mujeres, estas diferencias se pueden considerar clínicamente despreciables. (Tabla I)

Al realizar el análisis Estadístico de Concordancia de Bland \& Altman, este reveló que sistemáticamente la distancia OJO/TRAGUS sobre estima a la distancia OJO/OREJA, por lo que se procede a construir estas diferencias y establecer sus límites de confianza (Tabla III), encontrando concordancia entre ambas medidas solo en el grupo de los hombres, esto debido a que el intervalo de confianza del $95 \%$ de este grupo contiene el 0 , lo que quiere decir que existe un $95 \%$ de certeza que la diferencia del promedio de ambas medidas sean 0 , por ende concordantes. En el grupo total y en el de las mujeres se puede observar que dentro de los límites de confianza no está el cero, lo que indica que las distancias estadísticamente no son concordantes, sin embargo, como la diferencia promedio no alcanza a ser $1 \mathrm{~mm}$, estos resultados fueron analizados con la finalidad de conocer la magnitud de la sobre estimación (Tabla III), la cual se estimó con la ecuación de la recta, cuyo coeficiente de la pendiente determinó que en el grupo total la distancia clínica OJO/ TRAGUS sobre estima a la distancia OJO/OREJA en un $2,34 \%$ y en solo un $0,23 \%$ en el grupo de las mujeres. Con estos resultados y sabiendo que el método Craneométrico de Knebelman, es un método válido, es que podemos modificar el factor de ajuste de Knebelman con la finalidad de utilizar la distancia ángulo externo del ojo/surco tragus facial propuesta por Romo ${ }^{(8)}$ en la determinación de la DVO mediante el Método Craneométrico de Knebelman. Actualmente el ajuste proporcional de Knebelman construido en el diseño de su dispositivo establece que la distancia OJO/OREJA menos $4 \mathrm{~mm}$ es igual a la DVO, medida entre subnasal y la superficie inferior más anterior de la mandíbula. Por lo tanto, conociendo las concordancias y magnitudes de sobre estimación podemos proponer fórmulas para transformar la distancia clínica OJO/ TRAGUS a la distancia craneométrica OJO/OREJA de Knebelman (Tabla IV).

Tabla 4. Fórmula ideada para obtener la medida OJO/OREJA de Knebelman

\begin{tabular}{|c|c|c|c|}
\hline Grupo & Fórmula & $\begin{array}{c}\text { Factor de } \\
\text { Knebelman (FK) }\end{array}$ & \\
\hline Total & $\mathrm{Oj} / \mathrm{Tr}-(\mathrm{Oj} / \mathrm{Tr} \times \mathbf{0}, 0234)$ & $-4 \mathrm{~mm}$. & $=$ DVO \\
\hline Hombres & $\mathrm{Oj} / \mathrm{Tr}$ & $-4 \mathrm{~mm}$. & $=\mathrm{DVO}$ \\
\hline Mujeres & $\mathrm{Oj} / \mathrm{Tr}-(\mathrm{Oj} / \mathbf{T r} \times \mathbf{x} 0,0023)$ & $-4 \mathrm{~mm}$. & $=\mathrm{DVO}$ \\
\hline
\end{tabular}

Con la finalidad de simplificar el cálculo y hacer más sencillo el ejercicio de determinación de la DVO, se aplicó esta fórmula a los valores máximos y mínimos de la distancia OJO/TRAGUS obtenidos en el análisis estadístico descriptivo, de esta forma se obtuvo un factor de ajuste de Knebelman (FK) modificado máximo y mínimo para cada grupo (Tabla V).

Sabiendo que sistemáticamente la distancia OJO/TRAGUS sobre estima a la distancia OJO/OREJA, se realizó el promedio de estos factores de ajustes modificados obteniendo un valor de $4,61 \mathrm{~mm}$ el cual se aproximó a un número neto para su utilización con dispositivos milimetrados dejándolo en $5 \mathrm{~mm}$.

Al comparar nuestros resultados de la medición de la distancia facial ángulo externo del ojo al surco tragus facial con los obtenidos por Romo ${ }^{(8)}$ y Contreras ${ }^{(18)}$ podemos observar que son mayores a los de ellos, pero al aplicar el factor de Knebelman modificado, obtenemos valores de DVO bastante similares a los de Romo ${ }^{(8)}$, pero mayores a los de Contreras ${ }^{(18)}$, 
Tabla 5. Estimación FK modificado con valores máximos y mínimos del estudio

\begin{tabular}{|ccc|c|}
\hline Grupo & Fórmula aplicada & FK & FK modificado \\
\hline \multirow{2}{*}{ Total } & $\mathbf{8 0 . 6}-(\mathbf{1 . 8 8})$ & & $-5.88 \mathrm{~mm}$. \\
& $\mathbf{6 5 . 4}-(\mathbf{1 . 5 3})$ & $-4 \mathrm{~mm}$. & $-5.53 \mathrm{~mm}$. \\
\hline \multirow{2}{*}{ Hombres } & $\mathbf{8 0 . 6}$ & $-4 \mathrm{~mm}$. & $-4 \mathrm{~mm}$. \\
& 68.6 & & $-4 \mathrm{~mm}$. \\
\hline \multirow{2}{*}{ Mujeres } & $\mathbf{8 0 . 1}-(0.10)$ & $-4 \mathrm{~mm}$. & $-4.10 \mathrm{~mm}$. \\
& $\mathbf{6 5 . 4}-(0.15)$ & & $-4.15 \mathrm{~mm}$. \\
\hline
\end{tabular}

lo que se podría explicar por la diferencia étnica de las poblaciones estudiadas.

Sin embargo, al igual que Romo ${ }^{(8)}$ y Contreras ${ }^{(18)}$, podemos concluir que la medida facial propuesta, distancia OJO/TRAGUS puede ser utilizada para determinar la DVO, con la diferencia que no la consideramos equivalente sino que la ajustamos proporcionalmente restándole un factor y así determinar la distancia subnasal mentón, es decir la DVO, siguiendo lo establecido por Knebelman.

El estudio de la distancia OJO/TRAGUS y los resultados obtenidos en nuestra investigación nos permiten proponer una modificación al Método Craneométrico de Knebelman. Por lo tanto, y sabiendo que la Dimensión Vertical Oclusal(1,2) es la altura del segmento inferior de la cara cuando la mandíbula está en su posición de máxima intercuspidación, determinada entre dos puntos arbitrarios, uno en el maxilar y otro en la mandíbula, coincidentes con la línea media, podemos concluir que la distancia ángulo externo del ojo/surco tragus facial menos $5 \mathrm{~mm}$ puede ser usada para predecir la distancia subnasal mentón (zona más anterior e inferior) con razonable seguridad y de esta forma establecer la DVO.

\section{CONCLUSIONES}

Si bien no existe una concordancia perfecta entre la distancia craneométrica OJO/OREJA y la distancia clínica OJO/TRAGUS, las diferencias no representan significancia clínica y la evaluación de las sobre estimaciones nos permiten modificar el método Craneométrico de Knebelman para su aplicación clínica sin Craneometro, estableciendo que la distancia clínica surco tragus facial al ángulo externo del ojo medida con un pie de metro menos $5 \mathrm{~mm}$ nos permite predecir la distancia subnasal mentón (zona más anterior e inferior) con razonable seguridad y de esta forma establecer de la Dimensión Vertical Oclusal.

\section{CONFLICTO DE INTERÉS}

Los autores declaran no tener ningún conflicto de interés

\section{Bibliografía}

1.- Manns Arturo. Mecanismos neuromusculares centrales o cerebrales. En: Manns Arturo. Diaz Gabriela. Sistema Estomatognático. Chile. Ed. Ximpauser ; 1995. P 158-159.

2. - The Glossary of Prosthodontic Terms. J Prosthet Dent. 2005; 94:10-92.

3. - Turner KA, Missirlian DM. Restoration of the extremely worn dentition. J Prosthet Dent. 1984; 52:467-474.

4. - Mohindra NK, Bulman JS. The effect of increasing vertical dimension of occlusion on facial aesthetics. Br Dent J. 2002; 192:164-168.

5.- Coelho Goiato M, Micheline Dos Santos D, Vilela Sonego M. Abordagem. Clinical Approach of records used for restoration vertical dimension of occlusion prosthesis total. Rev Odontol Aracatuba. 2013; 34:45-49.

6.- García H, García J. Determinación de la dimensión vertical en el paciente desdentado. Rev Soc de Prot Est. 1994, 3 (1): 29-35.

7.- Gaete Baldi M., Riveros Reciné N. y Cabargas Morello J. Dimensión vertical oclusal (DVO): Análisis de un método para su determinación. Rev Dent Chile. 2003; 94(2): 17-21.

8.- Romo Ormazabal F, Jorquera Henríquez C, Irribarra Mengarelli R. Determinación de la dimensión vertical oclusal a través de la distancia clínica ángulo externo del ojo al surco tragus facial. Rev Dent Chile. 2009; 100:26-33.

9.- Gaete Saldaña P. Gaete Baldi M. Cáceres Ponce M. Evaluación craneométrica, utilizando el craneometro de Knebelman, de la dimensión vertical oclusal obtenida mediante un procedimiento clínico clásico. Rev Dent Chile. 2012; 103(2):29-35

10.- Quiroga del Pozo R, Riquelme Belmar R, Sierra Fuentes M, Del Pozo Bassi J, Quiroga Aravena R. Determinación de la dimensión vertical oclusal en desdentados totales: comparación de métodos convencionales con el craneometro de Knebelman. Rev Clin Periodoncia Implantol Rehabil Oral. 2012; 5:20- 24.
11. - Misch, C. Objective vs. subjective methods for determining vertical dimension of occlusion. Quintessence Int. 2000; 31(4): 280-82.

12. - Documento en internet: Knebelman Stanley. Method for determining vertical dimension. U.S. Patent No. 4718850. January 1988. [consultado 01/08/2018]. Disponible en: www.freepatentsonline.com/4718850.html.

13. - Chou TM, Moore DJ, Young L Jr, Glaros AG. A Diagnostic craniometric method for determining occlusal vertical dimension. J Prosthet Dent. Jun;71(6):568-574 1994.

14.- Delić Z, Simunović-Soskić M, Perinić-Grzić R, Vukovojac S, Rajić Z, Kuna T, Kuna T. Evaluation of craniometric methods for determination of vertical dimension of occlusion. Coll Antropol. Jul; 24 (Suppl 1): 31-35. 2000.

15. - Blaes, J. Pearls for you practice. Primera edición. Ed. PennWell Corporation. 2001:37.

16.- Cardoso S. Evaluación de la dimensión vertical oclusal mediante el método craneométrico de Knebelman en una población peruana con relación a biotipo facial. Tesis para optar al título profesional de Cirujano Dentista. Universidad Nacional Mayor de San Marcos. Lima, Perú. 2014.

17.- Quiroga del Pozo, R; Sierra Fuentes, M; del Pozo Bassi, J; Quiroga Aravena, R. Dimensión vertical oclusal: comparación de 2 métodos cefalométricos. Rev Clin Periodoncia Implantol Rehabil Oral. 2016; 9(3):264-270

18.- Contreras J. Evaluación de los métodos de Willis y Knebelman para determinar la dimensión vertical oclusal en pobladores de Taquile, Puno-2016. Tesis para optar al título profesional de Cirujano Dentista. Universidad Nacional del Altiplano. Facultad de Ciencias de las Salud. Escuela Profesional de Odontología. Puno, Perú. 2016. 